\title{
Formation of Single-atom-thick Copper Oxide Monolayers
}

Kuibo Yin ${ }^{1,2}$, Yu-Yang Zhang ${ }^{3,2}$, Yilong Zhou ${ }^{1}$, Litao Sun ${ }^{1,4}$, Matthew F. Chisholm², Sokrates T. Pantelides $^{3,2}$ and $\mathrm{Wu} \mathrm{Zhou}^{2}$

1. SEU-FEI Nano-Pico Center, Key Lab of MEMS of Ministry of Education, Southeast University, Nanjing 210096, China.

2. Materials Science \& Technology Division, Oak Ridge National Laboratory, Oak Ridge, TN 37831, USA.

3. Department of Physics and Astronomy and Department of Electrical Engineering and Computer Science, Vanderbilt University, Nashville, TN 37235, USA.

4. Center for Advanced Materials and Manufacture, Joint Research Institute of Southeast University and Monash University, Suzhou 215123, China

Two-dimensional (2D) crystals display many intriguing physical and chemical properties that are distinctly different from their bulk parent counterparts. A well-known example is the transition from indirect to direct band gap in monolayer $\mathrm{MoS}_{2}$ [1]. Among all 2D crystals, monolayer oxides are an interesting class of materials because of the coupled degrees of freedom (charge, spin, lattice) that are present in bulk oxides to which quantum confinement is now added. We can, therefore, expect a wide spectrum of electronic and magnetic properties with potential for applications. Many effects have been made to fabricate 2D oxides. However, as most of oxides are non-layered structures, the fabrication of 2D oxides has been limited [2-3]. Most published 2D oxide materials are either two to three atomic layers thick, as the exfoliated perovskites, or strongly bonded with the substrate. It is still elusive whether unsupported oxide monolayers can exist or not without the assistance of pore edge or substrate.

Bulk $\mathrm{CuO}$ and $\mathrm{Cu}_{2} \mathrm{O}$ have received a great deal of attention because of the similarity of $\mathrm{Cu}-\mathrm{O}$ bonding to the planar $\mathrm{Cu}-\mathrm{O}$ structures that give rise to high-temperature superconductivity in $\mathrm{Cu}$-based oxides. In addition, these materials are widely studied because of their importance in the fabrication of supported catalysts, gas sensors, earth abundant solar cells, thin-film transistors, and so on. Recently, a superior visible-light-conversion efficiency has been achieved in a four-atom-thick $\mathrm{Cu}_{2} \mathrm{O}$ film photoelectrode [4]. However, single-atom-thick copper oxide layers in isolation have not yet been fabricated.

In this study, we fabricated the single-atom-thick copper oxide monolayers using electron beam irradiation (as shown in Figure 1). Small clusters of copper oxide can form monolayer nanosheets with a square $\mathrm{Cu}$ sub-lattice under a $60-\mathrm{keV}$ electron beam irradiation, either spanning graphene pores to form unsupported monolayer oxide membranes or being supported on the graphene surface. Electron-energyloss spectroscopy results confirmed the exclusive presence of $\mathrm{Cu}$ and $\mathrm{O}$ element. Both $\mathrm{CuO}$ and $\mathrm{Cu}_{2} \mathrm{O}$ square monolayer are possible while $\mathrm{CuO}$ is more stable than $\mathrm{Cu}_{2} \mathrm{O}$ in energy, as demonstrated by quantum mechanical calculations. As the $\mathrm{Cu}-\mathrm{Cu}$ distance in the fabricated sample with a statistical analysis is more consistent with the calculated $\mathrm{Cu}-\mathrm{Cu}$ distance with the latest hybrid functional, we deduce that the as-fabricated sample is $\mathrm{CuO}$ monolayer. Free-standing $\mathrm{CuO}$ and $\mathrm{Cu}_{2} \mathrm{O}$ monolayer are stable as proved by our calculations. Their bandgaps are $\sim 3 \mathrm{eV}$. Moreover, $\mathrm{CuO}$ monolayer has an indirect bandgap while $\mathrm{Cu}_{2} \mathrm{O}$ monolayer has a direct one, which suggested that the electronic and optical properties of monolayer oxidized copper can be tuned by the oxygen content. 


\section{References:}

[1] Y. Zhang; T.-R. Chang; B. Zhou; Y.-T. Cui; H. Yan; Z. Liu; F. Schmitt; J. Lee; R. Moore; Y. Chen; H. Lin; H.-T. Jeng; S.-K. Mo; Z. Hussain; A. Bansil; Z.-X. Shen, Nat Nano 2014, 9, 111-115.

[2] R. Ma; T. Sasaki, Advanced Materials 2010, 22, 5082-5104.

[3] M. Osada; T. Sasaki, Advanced Materials 2012, 24, 210-228.

[4] S. Gao; Y. Sun; F. Lei; J. Liu; L. Liang; T. Li; B. Pan; J. Zhou; Y. Xie, Nano Energy 2014, 8, 205213.

[5] We thank Junhao Lin, Qian He, Miaofang Chi, Juan-Carlos Idrobo, and Andrew Lupini for useful discussions.
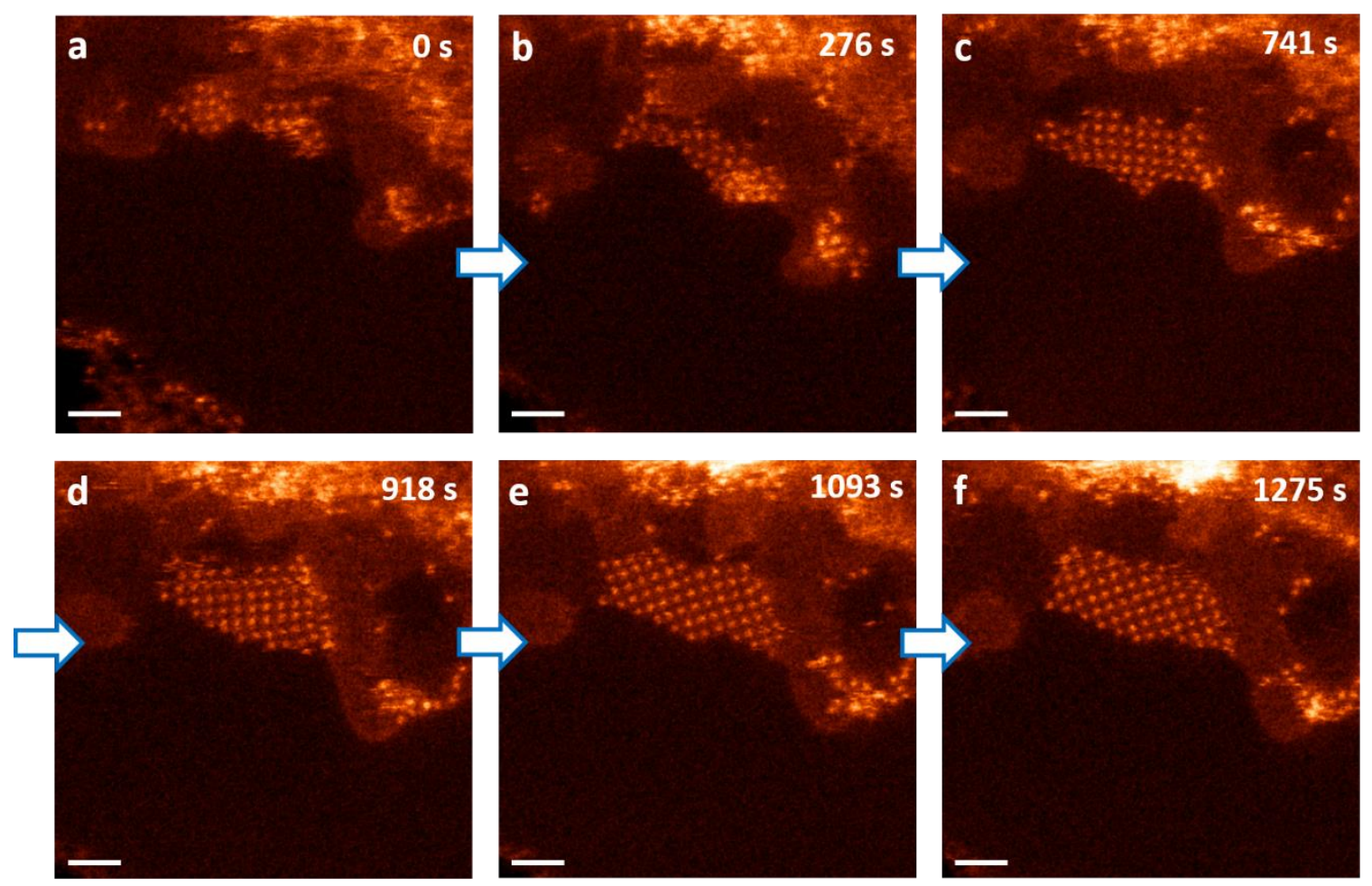

Figure 1. Formation process of a monolayer $\mathrm{CuO}$ on graphene substrate. (a)-(f) Time series of STEM$\mathrm{ADF}$ images, showing the migration of $\mathrm{CuO}$ and the formation of ordered monolayer structure on graphene surface. Scale bars: $1 \mathrm{~nm}$. 